\title{
Use of over-the-scope clips (OTSC) for hemostasis in gastro- intestinal bleeding in patients under antithrombotic therapy
}

\section{(ㄷ)(구)(우}

\author{
Authors \\ Institutions \\ 1 HELIOS Park-Klinikum Leipzig, Klinik für Innere Medizin \\ II, Leipzig, Germany \\ 2 Herzzentrum Leipzig, Klinik für Herzchirurgie, Leipzig, \\ Germany
}

Regina Lamberts ${ }^{1}$, Anna Koch ${ }^{1}$, Christian Binner ${ }^{2}$, Marcus Zachäus ${ }^{1}$, Ingrid Knigge ${ }^{1}$, Mark Bernhardt ${ }^{1}$, Ulrich Halm ${ }^{1}$

submitted 2.11 .2016

accepted after revision 2.2.2017

\author{
Bibliography \\ DOI https://doi.org/10.1055/s-0043-104860 | \\ Endoscopy International Open 2017; 05: E324-E330 \\ (c) Georg Thieme Verlag KG Stuttgart · New York \\ ISSN 2364-3722
}

Corresponding author

Prof. Dr. med. Regina Lamberts, HELIOS Park-Klinikum

Leipzig, Strümpellstrasse 41, D-04289 Leipzig

Fax: $+49 / 0341 / 8642264$

regina.lamberts@helios-kliniken.de

\section{ABSTRACT}

Background and study aims In patients taking different regimens of antithrombotic and/or anticoagulant therapy, endoscopic management of gastrointestinal bleeding represents a major challenge due to failing endogenous hemostasis. In this retrospective study we report on success rates with the over-the-scope clip (OTSC) system in upper and lower gastrointestinal bleeding in this highrisk patient population.

Patients and methods Between February 2011 and June 2014, 75 patients were treated with an OTSC for active gastrointestinal bleeding. Success rates with the first endoscopic therapy, rebleeding episodes, their management and the influence of antithrombotic or anticoagulant therapy were analyzed retrospectively.

Results Application of the OTSC resulted in immediate hemostasis (primary success rate) in all 75 patients. However, in $34.7 \%$ a rebleeding episode was noted that could be treated by further endoscopic interventions. Only 3 patients had to be sent to the operating room because of failure of endoscopic therapy. In the rebleeding group the use of antiplatelet therapies was higher $(73.1 \%$ vs. $48.9 \%$ ).

Conclusions Application of the OTSC in Gl bleeding results in a high rate of primary hemostasis. Rebleeding occurs in up to $35 \%$ of patients receiving antithrombotic/ anticoagulant therapy but can be managed successfully with further endoscopic treatments. Patients in the rebleeding group were more frequently treated with antiplatelet agents. Radiological or surgical therapy was reserved for a small subgroup not successfully managed by repeated endoscopic therapies. OTSC application is the treatment of choice in high-risk patients when conventional clips used as first-line treatment fail.

\section{Introduction}

The medical therapy of upper gastrointestinal bleeding significantly improved during the last 30 years due to the development of effective suppression of gastric acid secretion by proton pump inhibitors. In addition the eradication of helicobacter pylori has decreased incidence rates of gastric and duodenal ulcers [1]. Although the number of hospitalizations due to peptic ulcer disease has decreased during the last decades [2], in contrast to this nationwide trend, admission rates for gastric and duodenal ulcer hemorrhage and perforation in older patients even increased [3] and mortality rates remained essentially unchanged at $5 \%$ to $8 \%$ [4]. This may in part be due to the increasing and intensive use of antithrombotic and/or anticoagulant medications for treatment of various cardiac, vascular or circulatory disorders in a consistently older population of patients with multiple morbidities $[5,6]$. This population represents a high-risk group with increased bleeding and higher mortality rates between $10 \%$ and $20 \%$. Only few data are available concerning rebleeding rates in this special situation [4].

The successful treatment of upper and lower gastrointestinal bleeding in this patient population represents a major challenge for gastroenterologists due to a non-functioning endogenous hemostasis. First, individual risk calculation is difficult due to the fact that medical therapy is not reflected in pathologic laboratory results (as in the case of novel oral anticoagulants (NOAC). Second, antithrombotic therapy often cannot be stopped due to shortly performed catheter interventions. In 
that situation secure, easily performed and highly successful treatment of bleeding vessels is necessary to reduce further risks of therapeutic failures.

In the last decade, a novel over-the-scope clip system (OTSC) with high success rates has been introduced in endoscopic therapy of gastrointestinal bleeding or closure of gastrointestinal fistula/perforations [7-10]. The clip is easily attached to the tip of the scope and can be readily released at the site of the bleeding using a procedure similar to ligation of esophageal varices. No special endoscopic training is required for successful use of the system. Primary success rates between $78 \%$ and $100 \%$ have been reported, however, they are mostly in small patient series with varying indications for therapy [7-10].

In this retrospective study we analyzed the success rate with OTSC for hemostasis in gastrointestinal bleeding in 75 patients treated in our gastroenterological department between February 2011 and June 2014. The patients presented with upper or lower gastrointestinal bleeding and in more than $75 \%$, it was in the setting of antithrombotic therapy. Besides primary success rates we analyzed rebleeding episodes with respect to use of antithrombotic and/or anticoagulant medications at the time of first endoscopic intervention. This study focuses on use of OTSC in gastrointestinal bleeding only in the largest patient group reported in the literature so far.

\section{Patients and methods}

From February 2011 to June 201475 patients with acute upper or lower gastrointestinal bleeding treated endoscopically by OTSC application were included in this study. Endoscopic interventions were performed by expert gastroenterologists in 65 cases $(87 \%)$ and in $10(13 \%)$ by in-training endoscopists, respectively. We performed a retrospective evaluation of the therapeutic use of OTSC, which was disclosed to the local ethic committee. Patient characteristics are reported in $>$ Table 1 in detail. Time, diagnosis and therapy of the first and all follow-up endoscopic examinations (location and type of lesions, Forrest classification) were extracted from patient records. In addition the presence and type of oral or intravenous (IV) antithrombotic therapy at the time of initial bleeding as well as at the time of rebleeding episodes were analyzed with respect to success of the endoscopic therapy. Values are given as means. Comparisons between different patient groups were done by Fisher's exact test employing the SPSS software version 18.0.

\section{Results}

The 75 patients included 55 men (73.3\%) and 20 women $(26.7 \%)$. They represented a mixed population from our departments of gastroenterology and abdominal surgery $(n=$ $47)$ and the Heart Centre Leipzig $(n=28)$. Mean age was 71.7 years (range 45-92). At the index gastroscopy the bleeding source was localized in the upper gastrointestinal tract in $90.7 \%(n=68)$. In 7 patients $(9.3 \%)$ bleeding was seen in the lower gastrointestinal tract (colon $[n=4]$ or rectum $[n=3]$ ). In the upper gastrointestinal tract, duodenal ulcers were the most frequent bleeding cause, followed by the stomach and
- Table 1 Patients characteristics of the total study group $(\mathrm{n}=75)$

\begin{tabular}{|c|c|c|}
\hline Characteristic & $\mathbf{n}$ & $\%$ \\
\hline Men/women & $55 / 20$ & $73.3 / 26.7$ \\
\hline Mean age (years) & 71.7 (range 45-92) & \\
\hline Active bleeding source & & 90.7 \\
\hline - Upper Gl tract & $68 / 75$ & 4.0 \\
\hline - Esophagus & $3 / 75$ & 29.3 \\
\hline - Stomach & $22 / 75$ & 57.3 \\
\hline - Duodenum & $43 / 75$ & 9.3 \\
\hline - Lower Gl tract & $7 / 75$ & 5.3 \\
\hline - Colon & $4 / 75$ & 4.0 \\
\hline - Rectum & $3 / 75$ & \\
\hline
\end{tabular}

Type of lesion

\begin{tabular}{|l|l|l}
\hline - Ulcer & $62 / 75$ & 82.7 \\
\hline
\end{tabular}

\begin{tabular}{|l|l|l|}
\hline - Postinterventional & $10 / 75$ & 13.3 \\
\hline
\end{tabular}

\begin{tabular}{l|l|l} 
- Varia $^{2}$ & $3 / 75$ & 4.0 \\
\hline
\end{tabular}

Bleeding source in upper $\mathrm{Gl}$ tract

\begin{tabular}{|l|l|l|}
\hline - Duodenal ulcer & $41 / 62$ & 66.1 \\
\hline
\end{tabular}

\begin{tabular}{|l|l|l|}
\hline - Gastric ulcer & $18 / 62$ & 29.0 \\
\hline
\end{tabular}

Forrest classification

\begin{tabular}{|r|r|r|}
\hline - Ia & $18 / 75$ & 24.0 \\
\hline - Ib & $33 / 75$ & 44.0 \\
\hline - Ila & $22 / 75$ & 29.3 \\
\hline - Ilb & $2 / 75$ & 2.7 \\
\hline
\end{tabular}

Antithrombotic therapy

\begin{tabular}{|c|c|c|}
\hline No therapy & $18 / 75$ & 24.0 \\
\hline Antiplatelet therapy & $44 / 75$ & 58.7 \\
\hline - Antiplatelet monotherapy & $34 / 75$ & 45.8 \\
\hline - Aspirin & $26 / 75$ & 34.7 \\
\hline - Clopidogrel & $8 / 75$ & 10.7 \\
\hline $\begin{array}{l}\text { - Dual antiplatelet therapy } \\
\text { (DAPT) }\end{array}$ & $10 / 75$ & 13.3 \\
\hline $\begin{array}{l}\text { Inhibitors of plasmatic } \\
\text { coagulation }\end{array}$ & $13 / 75$ & 17.3 \\
\hline Combination therapy ${ }^{3}$ & $12 / 75$ & 16.0 \\
\hline \multicolumn{3}{|c|}{$\begin{array}{l}\text { Gl, gastrointestinal; EMR, endoscopic mucosal resection } \\
1 \text { surgery, polypectomy, PEG-removal, EMR; } \\
2 \text { Mallory Weiss lesion, diverticular bleeding; } \\
{ }^{3} \text { patients with combination therapy are also included in the "antiplatelet } \\
\text { therapy" and "inhibitors of plasmatic coagulation" group }\end{array}$} \\
\hline
\end{tabular}

the esophagus ( $\triangleright$ Table 1 ). In the colon, we observed 2 diverticular bleeds, 1 post-polypectomy bleed (close to the right flexure) and 1 ulcer bleed in the cecum after preceding argon plasma coagulation (APC) therapy of angiectasia. 
Ulcers were the most frequent causes of bleeding (82.7\%), followed by post-interventional causes as polypectomy $(n=3)$, endoscopic mucosal resection (EMR) $(n=1)$, rectal surgery $(n=$ $1)$, percutaneous enteral gastrostomy (PEG) removal $(n=2)$ and bleeding at surgical anastomoses $(n=3)$, representing all together $13.5 \%$. Other causes (Mallory Weiss lesion, diverticular bleeding) were rare ( $\$$ Table $\mathbf{1})$.

The Forrest classification of bleeding at the index endoscopy is also shown in $>$ Table 1 , indicating active bleeding (Forrest la and $b)$ in $68 \%(n=51)$ of patients.

OTSC was the primary endoscopic treatment in 44 (58.7\%) patients, in 31 patients (41.3\%) it was used after preceding endoscopies without long-lasting hemostasis. Previous endoscopic attempts to achieve hemostasis included through-thescope (TTS) clip application in 30 cases (40\%, > Fig.1), combined with adrenalin injection in 10 patients and fibrin glue injection as well as APC therapy in 1 patient each. The traumatic (type t) OTSC (12/6t) was used almost exclusively with the therapeutic gastroscope; in case of colonic bleeding we used a size 14/6t. An anchor (to pull the tissue into the cap of the clip) as well as the traumatic gc variant of the clip (long branches) were applied only once each.

OTSC resulted in immediate cessation of bleeding in $100 \%$ of patients except that in 1 patient, incomplete adaptation of wound edges of a large gastric ulcer required additional application of a TTS clip in the periphery. However, we observed a significant number of rebleeding episodes during regularly scheduled or emergency re-endoscopies in 26 patients (34.7\%).

Patients with rebleeding episodes represented a critically ill population with a high rate of coronary heart disease $(n=14$, $53.8 \%$ ), recent cardiac/vascular or gastrointestinal surgery ( $n=11,42.3 \%$ ), recent or present mechanical ventilation ( $n=11,42.3 \%$ ), septicemia or systemic inflammatory response syndrome $(n=8,30.8 \%)$, diabetes mellitus $(n=9,34.6 \%)$, and/ or carcinoma history $(n=4,15.4 \%)$.

Rebleeding was seen in a similar proportion in patients with gastric $(42.3 \%)$ or duodenal ulcer (50\%) and was observed almost exclusively in the upper gastrointestinal tract (92.3\%). Rebleeding was present in $65 \%$ of patients during the first reendoscopy. The criteria for re-endoscopy were not constant throughout the study period. In the beginning, re-endoscopy was routinely scheduled within the first 24 to 48 hours after initial endoscopy. Due to increasing experience, in the latter part of the study, re-endoscopy was mainly done in cases of clinical suspicion of rebleeding (e.g. continuous transfusion requirements, hematemesis or melena, hemodynamic instability, etc.). Thus, indication for re-endoscopy was mainly based on the clinical judgement of the physician taking care of the patient. The time interval from index endoscopy to endoscopically verified rebleeding varied between 0 and 8 days (mean 2.6 days), only 3 patients showed rather late rebleeding from the same source after 17,30 , and 45 days respectively.

The number of follow-up endoscopies $(n=112)$ varied from 0 to 6 , with a mean number of 1.5 /patient (no control in 15 , 1 control in 32, 2 controls in 15, 3 controls in 5, 4 controls in 6 , and 5 and 6 controls in 1 patient each).

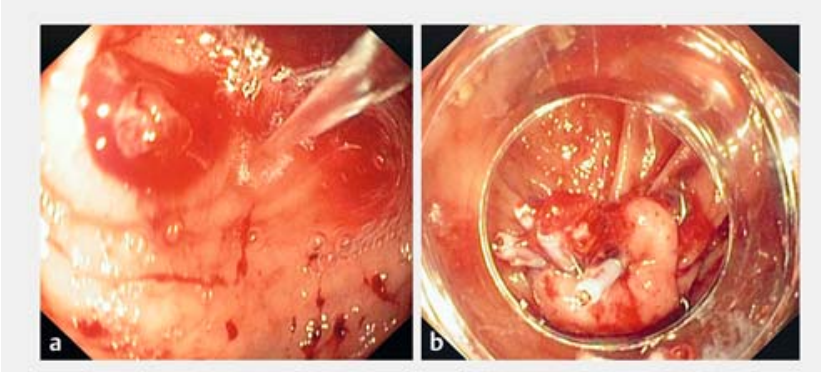

- Fig. 1 a Forrest Ib bleeding from a cecal ulcer seen during water jet irrigation. b Successful OTSC application after failed attempts of treatment with TTS clips.

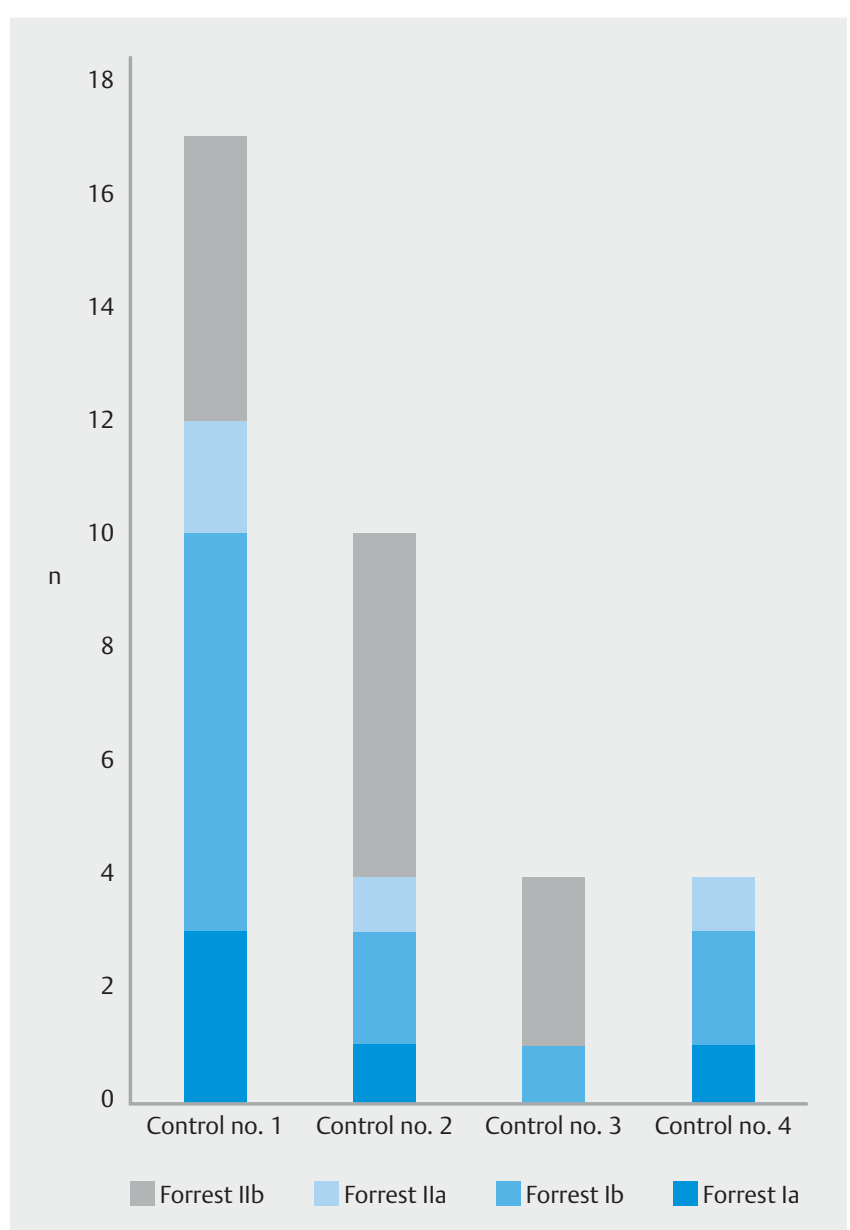

- Fig. 2 Forrest classification of rebleeding episodes in control endoscopies no. $1-4$.

Rebleeding episodes were evaluated separately for each control endoscopy according to the Forrest classification ( $\triangleright$ Fig.2). Besides the decreasing number of bleeding episodes at control endoscopies the severity of bleeding according to the Forrest classification decreased steadily from the first to the last control endoscopy.

Patients with increasing Forrest classifications in comparison to previous endoscopies as well as late rebleeders were at high 
- Table 2 Comparison of clinically relevant data of patients with or without rebleeding episodes after OTSC application.

\begin{tabular}{|c|c|c|c|c|}
\hline & \multicolumn{2}{|c|}{ Rebleeding $(n=26)$} & \multicolumn{2}{|c|}{ No rebleeding $(n=49)$} \\
\hline & n & $\%$ & $\mathbf{n}$ & $\%$ \\
\hline \multicolumn{5}{|l|}{ Active bleeding source } \\
\hline - Upper GI tract & $24 / 26$ & 92.3 & $44 / 49$ & 89.8 \\
\hline - Lower GI tract & $2 / 26$ & 7.7 & $5 / 49$ & 10.2 \\
\hline \multicolumn{5}{|l|}{ Ulcer location } \\
\hline - Duodenal ulcer & $13 / 26$ & 50.0 & $30 / 49$ & 61.2 \\
\hline - Gastric ulcer & $11 / 26$ & 42.3 & $11 / 49$ & 22.4 \\
\hline \multicolumn{5}{|l|}{ Forrest classification } \\
\hline - la & $10 / 26$ & 38.5 & $8 / 49$ & 16.3 \\
\hline . Ib & $9 / 26$ & 34.6 & $24 / 49$ & 48.9 \\
\hline - Ila & $7 / 26$ & 26.9 & $15 / 49$ & 30.6 \\
\hline - IIb & $0 / 26$ & & $2 / 49$ & 4.1 \\
\hline \multicolumn{5}{|l|}{ Antithrombotic therapy } \\
\hline No therapy & $4 / 26$ & 15.4 & $14 / 49$ & 28.6 \\
\hline Antiplatelet therapy & $19 / 26$ & 73.1 & $25 / 49$ & 51.0 \\
\hline - Monotherapy & $15 / 26$ & 57.7 & $19 / 49$ & 38.8 \\
\hline - Aspirin & $11 / 26$ & 42.3 & $15 / 49$ & 30.6 \\
\hline - Clopidogrel & $4 / 26$ & 15.4 & $4 / 49$ & 8.2 \\
\hline - Dual antiplatelet therapy (DAPT) & $4 / 26$ & 15.4 & $6 / 49$ & 12.2 \\
\hline Inhibitors of plasmatic coagulation & $3 / 26$ & 11.5 & $10 / 49$ & 20.4 \\
\hline Combination therapy ${ }^{1}$ & $5 / 26$ & 19.2 & $7 / 49$ & 14.3 \\
\hline
\end{tabular}

risk for surgery: 1 patient with a Forrest la bleed due to an ulcus Dieulafoy in the gastric fundus (at the forth control endoscopy 7.7 days after the index endoscopy) was scheduled for gastrectomy after repeated bleeding despite 2 additional OTSC applications, 2 laparotomies, coiling of the arteria gastrica sinistra and histoacryl injection therapy. The second patient with a late Forrest Ib duodenal ulcer bleed 6 days after index gastroscopy had shown lower Forrest classifications in 2 interval gastroscopies which didn't respond to various additional endoscopic therapies (application of a TTS clip, fibrin glue injection and application of a second OTSC after detachment of the first one). He was scheduled for surgery and surgical ligation of the arteria gastroduodenalis. The third critically patient with a Forrest Ib bleed due to a gastric ulcer 55 days after the first intervention was also scheduled for gastrectomy due to failure of the endoscopic therapy.

In the rebleeding group we observed a loss of the OTSC in 7 patients after a median of 5 days (range 3 to 55 days). No complete data are available for the non-rebleeding group due to missing control endoscopies. Early loss of the OTSC was asso- ciated with acute rebleeding (Forrest Ib) in only 1 patient. However, all 3 patients who had to be operated on because of failure of endoscopic therapy had lost the OTSC at various points in time before (days 4 to 55 ).

Rebleeding was seen from the center of the OTSC (adherent thrombus or bleeding/non-bleeding vessel) and the periphery (close to the teeth of the OTSC) in 13/12 patients each. Therapy in case of rebleeding was left to the decision of the endoscopist and included additional TTS clip application, fibrin glue injection, application of another OTSC, histoacryl and/or adrenalin injection (in decreasing order or combinations), or no intervention.

Rebleeding episodes were evaluated according to use of the OTSC as first- or second-line treatment. Rebleeding in the group of first-line OTSC was $28.9 \%$ (13/45) compared to $43.3 \%(13 / 30)$ in the group of second-line OTSC. In patients without rebleeding $65.3 \%$ had the OTSC as first-line treatment vs. $34.6 \%$ as secondline treatment; in the rebleeding group the ratio was $50 \% / 50 \%$.

Use of antithrombotic and/or anticoagulant therapy in the total patient group is shown in $>$ Table 1 . Antiplatelet therapy 
included use of aspirin and clopidogrel, either alone or in combination (dual antiplatelet therapy-DAPT), inhibition of plasmatic coagulation was achieved by either IV heparin or phenprocoumon. The percentage of patients receiving different medications in the rebleeding group vs. the non-rebleeding group is shown in > Table 2 . There was a trend toward more frequent use of antiplatelet therapy in the rebleeding group (73.1\%) vs. the non-rebleeeding group (51.0\%), however, that difference did not reach statistical significance $(P=0.086)$. In contrast, inhibitors of plasmatic coagulation (11.3\% vs. $20.4 \%$ ) as well as no therapy at all (15.4\% vs. $28.6 \%)$ were seen less frequently. Use of antiplatelet therapy is further illustrated in - Fig. 3, which shows higher percentages of aspirin (42.3\% vs. $30.6 \%)$ or clopidogrel (15.4\% vs. $8.2 \%$ ) as well as of dual therapy $(15.4 \%$ vs. $12.2 \%)$ in the rebleeding group.

At the time of rebleeding the antithrombotic therapy had been reduced in most patients: antiplatelet monotherapy had been stopped in 12 of 14 patients and partially been replaced by IV heparin. No patient received a dual platelet aggregation therapy (DAPT) any longer.

Our study group included 13 patients who required extracorporal circulation (either left ventricular assist device [LVAD] or extracorporal membrane oxygenation therapy [ECMO]) with an inherent need for intense anticoagulation to prevent clotting of the system. Interestingly, rebleeding episodes were not significantly different in patients with or without an extracorporal circulation $(38.5 \%$ vs. $33.9 \%, P>0.1)$.

By January 2015, 9 patients had died, 8 due to underlying cardiac disease and 1 due to a carcinoma history. No patient died due to early or late complications of the gastrointestinal bleeding episode.

\section{Discussion}

The first reports on use of the OTSC system in gastrointestinal endoscopy date back to 2007 [7]. Since then the system has been successfully used mainly for closure of gastrointestinal fistula and perforations as well as for bleeding vessels, especially in larger or fibrotic ulcers at difficult anatomic locations. Most published results refer to a mixed population with a small number of patients and different indications for use of the OTSC [611]. In this report we focused on use of the OTSC system for hemostasis in upper and lower gastrointestinal tract bleeding in the largest group of patients reported in the literature so far. We treated a mixed patient population from our departments of internal medicine and gastroenterology, abdominal surgery and the Heart Centre Leipzig. Because of the relatively high mean age of 71.7 years, the high proportion of patients receiving some kind of antithrombotic/anticoagulant therapy (76\%) and the frequent multiple morbidities, these patients belong to a high-risk group both with regard to bleeding rates as well as to rebleeding events, which according to the literature significantly increase short-term mortality $[5,6]$.

Overrepresentation of patients with upper gastrointestinal bleeding in our study is probably due to the fact that lower gastrointestinal bleeding (mostly bleeding from diverticula or angiectasia) has a high rate of spontaneous remission or can be

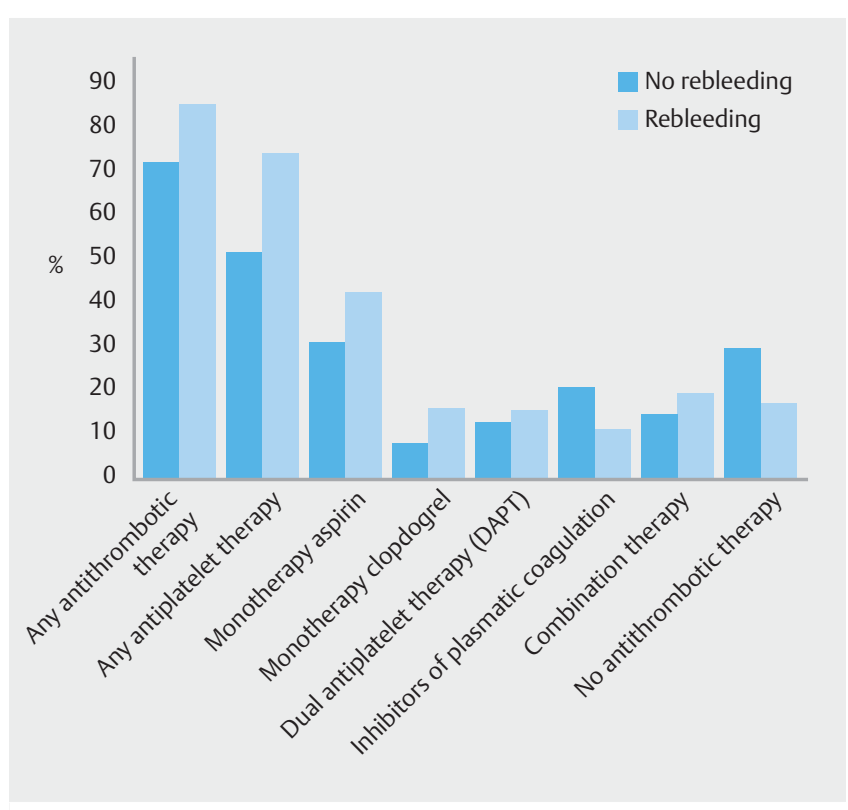

Fig. 3 Comparison of frequency and kind of antithrombotic therapies in patients with or without rebleeding after OTSC application.

treated by conventional methods (e. g. TTS clip, argon plasma coagulation) successfully. A third group of patients included in this study $(n=10)$ presented with acute bleeding episodes due to a previous endoscopic or surgical therapy. In view of the increasing invasiveness of endoscopic procedures, that group can be expected to increase in the future.

In case of large fibrotic ulcers, large bleeding vessels or difficult anatomic locations, duodenal bulb TTS clip therapy often requires application of several clips with a primary failure rate of up to $10 \%$ to $20 \%$ and a high rate of rebleeding episodes (15\%) [12]. Indeed, more than $40 \%$ of our patients had been treated with conventional methods such as TTS clip application, fibrin glue or adrenaline injection without long-lasting success before OTSC application. The effectiveness of OTSC placement as a rescue method for severe upper gastrointestinal bleeding has been shown in several studies that reported a $57 \%$ to $100 \%$ primary success rate with rebleeding rates between $7.4 \%$ and $42.8 \%$ [12 - 16]. In contrast to use as a rescue method, 2 studies reported on lower or even no rebleeding episodes if the OTSC was used as first-line endoscopic treatment in patients with high-risk upper gastrointestinal bleeding $[17,18]$. In accordance with work by Richter-Schrag et al. [18], our data also support the idea of more efficient treatment of bleeding episodes in high-risk patients when OTSC is used as primary therapy.

Application of OTSC in our hands has a high primary success rate $(100 \%)$ due to effective compression of the bleeding vessel. However, a rebleeding rate of almost $35 \%$ in our study was surprising to us because it is no better than data on the TTS clip and still far from satisfactory. In a study of patients in Japan, Ogasawara et al. [4] defined predictive factors for rebleeding in 69 out of 354 patients (19\%) which included age $>70$ years, shock on admission, hemoglobin $<8 \mathrm{mg} / \mathrm{dL}$, serum albumin $<3.3 \mathrm{~g} / \mathrm{dL}$, exposed vessels with a diameter $>2 \mathrm{~mm}$ and Forrest 
Type la and Ib bleeding. These criteria were present alone or in combination in a high percentage of our patients. In addition, as stated by Abraham [19], endoscopic hemostasis should be considered an activity that carries with itself a high risk of post-procedural bleeding of at least $5 \%$. Because the rebleeding episodes in our study were seen during a long time interval between 0 and 45 days, routine re-endoscopy at a fixed time point after application of OTSC does not seem to be justified.

It remains to be seen whether the high rate of rebleeding episodes in our study is due to the fact that about $75 \%$ of our patients used some kind of antithrombotic therapy. Interestingly, during the period covered by our study, none of the patients were treated with any NOACs which will probably further increase the problem in the future. Due to the fact that use of these drugs is not reflected in distinct laboratory parameters, it is difficult to calculate the risk for an individual patient (even in case of overdosing). In addition, use of antiplatelet agents is not reflected in routine laboratory parameters either. Therefore, despite differences especially in use of antiplatelet agents in patients with or without rebleeding episodes, this difference is not reflected in different coagulation parameters at the time of the index or follow-up endoscopies. Due to the complex patient histories with multiple diagnoses and extensive use of drug therapy, it was almost impossible to correlate individual laboratory parameters such as hemoglobin values, thrombocytes counts, or transfusion requirements with the gastrointestinal bleeding episode itself. Therefore, these data were not investigated further in this study.

The obviously missing influence of intense therapy with inhibitors of plasmatic coagulation (as in the case of extracorporeal circulation) on rebleeding as well as the trend to an increased frequency of antiplatelet therapies in the rebleeding vs. the non-rebleeding group points to the important influence of antiplatelet agents on risk of rebleeding in gastrointestinal peptic ulcer disease.

It has been shown in the literature that rebleeding episodes after a first endoscopic intervention significantly influence clinical outcome and mortality in these patients. In that respect, it is important to note that a high number of our patients were successfully managed with repeated endoscopic interventions. After application of an OTSC in the first place, these mostly included conventional methods such as TTS clips or injection therapy due to the often limited space for application of a second OTSC. The effect of repeated endoscopic interventions is reflected in the decreasing frequency of active rebleeding episodes (Forrest la and lb) during follow-up endoscopies. The favorable outcome of repeated endoscopic interventions in comparison to surgery in rebleeding after initial endoscopic bleeding control was also been reported in the pre-OTSC era [20] and should be considered especially in management of seriously ill patients who have a high number of comorbid conditions and are at increased intraoperative and postoperative risk. However, if there is an increase in Forrest classification grade after repeat endoscopies, these patients are candidates for alternative methods such as embolization, surgical ligation of the corresponding vessel or resection of the bleeding source. Indeed, in some patients all of the available techniques had to be of- fered depending on presence of comorbidities, risk of surgery or availability of techniques in an emergency situation. Only about $4 \%$ of our patients (4/75) finally required surgery and an emergency surgery rate of $6.5 \%$ to $7.5 \%$ has been reported in previous studies [21]. This is in contrast to the higher rate of surgical interventions (12.7\%) reported by Wedi et Hochberger [22] in patients receiving anticoagulants who were treated primarily with the OTSC for severe gastrointestinal bleeding, a group that is probably comparable to the patient population described here.

\section{Conclusion}

In conclusion, in the setting of acute gastrointestinal bleeding, OTSC is effective in achieving primary hemostasis in $100 \%$ of patients, even after pretreatment with conventional endoscopic techniques. This primary success rate is independent of the antithrombotic therapy used in individual patients. The high rate of primary hemostasis is in contrast to a rebleeding rate of $35 \%$, which is probably influenced by multiple factors including optimal clip application, medical therapy (especially ongoing antiplatelet agents) and the severity of the underlying disease. In a critically ill patient population primary use of OTSC reduces rebleeding events and should be the therapy of choice. However, also in this patient population, repeated endoscopic treatments to achieve definitive hemostasis may be justified and show promising results. Alternative techniques such as embolization or surgery remain rescue methods for a small subgroup of patients in whom repeated endoscopic therapies fail.

\section{Competing interests}

None

\section{References}

[1] Post PN, Kuipers EJ, Meijer GA. Declining incidence of peptic ulcer but not of its complications: a nation-wide study in the Netherlands. Aliment Pharmacol Ther 2006; 23: 1587-1593

[2] Wu CY, Wu CH, Wu MS et al. A nationwide population-based cohort study shows reduced hospitalizations for peptic ulcer disease associated with h. pylori eradication and proton pump inhibitor use. Clin Gastroenterol 2009; 7: 427-431

[3] Higham J, Kang JY, Majeed A. Recent trends in admissions and mortality due to peptc ulcer in England: increasing frequency of haemorrhage among elder subjects. Gut 2002; 50: 460 - 464

[4] Ogasawara N, Mizuno M, Masui R et al. Predictive factors for intractability to endoscopic hemostasis in the treatment of bleeding gastroduodenal peptic ulcers in Japanese patients. Clin Endosc 2014; 47: $162-173$

[5] Abraham NS, Hartmann C, Richardson P et al. Risk of lower and upper gastrointestinal bleeding, transfusions, and hospitalizations with complex antithrombotic therapy in elderly patients. Circulation 2013; 128: $1869-1877$

[6] Holster IL, Valkhoff VE, Kuipers E] et al. New oral anticoagulants increase risk for gastrointestinal bleeding: a systematic review and meta-analysis. Gastroenterology 2013; 145: 105 - 112 
[7] Kirschniak A, Kratt T, Stüker D et al. A new endoscopic over-thescope clip system for treatment of lesions and bleeding in the $\mathrm{Gl}$ tract: first clinical experiences. Gastrointest Endosc 2007; 66: 162 167

[8] Repici A, Arezzo A, De Caro G et al. Clinical experience with a new endoscopic over-the-scope clip system for use in the GI tract. Dig Liver Dis 2009; 41: 406-410

[9] Kirschniak A, Subutova N, Zieker D et al. The over-the-scope clip (OTSC) for the treatment of gastrointestinal bleeding, perforations and fistulas. Surg Endosc 2011; 25: 2901 - 2905

[10] Baron TH, Wong Kee Song LM et al. Use of an over-the-scope clipping device: multicenter retrospective results of the first U.S. experience (with videos). Gastrointest Endosc 2012; 76: 202-208

[11] Nishiyama N, Mori H, Kobara H et al. Efficacy and safety of the overthe-scope clip: including complications after endoscopic submucosal dissection. World J Gastroenterol 2013; 19: 2752-2760

[12] Wong SH, Sung JJY. Management of patients with rebleeding. Gastrointest Endosc Clin N Am 2015; 25: 569- 581

[13] Albert JG, Friedrich-Rust M, Woeste $G$ et al. Benefit of a clipping device in use in intestinal bleeding and intestinal leakage. Gastrointest Endosc 2011; 74: 389-397

[14] Manta R, Galloro G, Mangiavillano B et al. Over-the-scope clip (OTSC) represents an effective endoscopic treatment for acute GI bleeding after failure of conventional techniques. Surg Endosc 2013; 27: $3162-3164$

[15] Chan SM, Chiu PWY, Teoh AYB et al. Use of the over-the-scope clip for treatment of refractory upper gastrointestinal bleeding: a case series. Endoscopy 2014; 46: 428-431
[16] Skinner M, Gutierrez JP, Neumann H et al. Over-the-scope clip placement is effective rescue therapy for severe acute upper gastrointestinal bleeding. Endosc Int Open 2014; 2: E37-E40

[17] Manno M, Mangiafico S, Caruso A et al. First-line endoscopic treatment with OTSC in patients with high-risk non-variceal upper gastrointestinal bleeding; preliminary experience in 40 cases. Surg Endosc 2016; 30: 2026-2029

[18] Richter-Schrag J, Glatz T, Walker C et al. First-line endoscopic treatment with over-the-scope clips significantly improves the primary failure and rebleeding rates in high-risk gastrointestinal bleeding: A single-center experience with 100 cases. World J Gastroenterol 2016; 22: $9162-9171$

[19] Abraham NS. Management of antiplatelet agents and anticoagulants in patients with gastrointestinal bleeding. Gastrointest Endosc Clin N Am 2015; 25: 449-462

[20] Lau JYW, Sung JJY, Lam YH et al. Endoscopic retreatment compared with surgery in patients with recurrent bleeding after initial endoscopic control of bleeding ulcers. N Engl J Med 1999; 340: 751 - 756

[21] Abe N, Takeuchi $\mathrm{H}$, Yanagida $\mathrm{O}$ et al. Surgical indications and procedures for bleeding peptic ulcer. Dig Endoscopy 2010; 22: (Suppl. 01): $35-37$

[22] Wedi E, Sportes A, Hochberger JH. Endoscopic treatment of severe upper Gl-bleeding in anticoagulated patients using the over-thescope-clip (OTSC) - are we entering a new era. Gastrointestinal Endosc 2015; 81: AB448 